\title{
ANÁLISE DE ESTRATÉGIAS DE ISOLAMENTO SOCIAL PARA O ENFRENTAMENTO DA PANDEMIA DA COVID-19 EM MINAS GERAIS/BRASIL
}

\author{
A. O. CARDOSO ${ }^{1}$, N. G. SOUSA ${ }^{2}$, R.F. CARDOSO ${ }^{3}$, A.G. UTSUMI ${ }^{4}$ \\ Universidade Federal dos Vales do Jequitinhonha e Mucuri ${ }^{1}$, Universidade Federal do Triangulo Mineiro ${ }^{2,4}$ \\ Instituto Lucano ${ }^{3}$ \\ Orcid ID: https://orcid.org/0000-0002-4518-075X ${ }^{1}$ \\ anamaria.cardoso@ict.ufvjm.edu.br ${ }^{1}$
}

Submetido 27/10/2020 - Aceito 11/01/2021

DOI: $10.15628 /$ holos. 2020.11460

\section{RESUMO}

A pandemia da COVID-19 tem desafiado gestores e pesquisadores na busca de melhores estratégias para enfrentamento da doença e impedir o colapso do sistema de saúde. Este artigo avalia diferentes estratégias de isolamento social a fim de verificar sua eficácia na redução do número de infectados e mortes provenientes da COVID-19. Através da modelagem matemática e simulação computacional, analisou-se três diferentes tipos de isolamento social para o estado de Minas Gerais/Brasil, considerando o isolamento social rígido definido com uma redução de $80 \%$ do contato social, distribuídos em períodos ininterruptos de 48 (quarenta e oito) dias ou intercalados com períodos de isolamento social otimizado, buscando assim alternativas que viabilização a prestação de serviço e a geração de renda. A estratégia na qual adota-se o isolamento rígido apenas aos finais de semana, intercalando com o isolamento otimizado com redução de $55 \%$ do contato social de segunda a sexta, apresentou uma taxa de redução de $18,6 \%$ no número total de óbitos e infectados quando comparada com o mesmo período de isolamento rígido aplicado de forma ininterrupta, reduzindo em 8,6\% destes números em relação ao que se projeta hoje para o estado caso seja mantida a taxa média de isolamento atual. Os resultados obtidos permitem uma projeção do cenário da pandemia no estado e auxiliam na análise de alternativas para o enfrentamento desta em Minas Gerais e no Brasil.

PALAVRAS-CHAVE: modelagem matemática, coronavírus, quarentena, curva epidêmica

\section{ANALYSIS OF SOCIAL ISOLATION STRATEGIES FOR CONTROLING THE COVID-19 PANDEMIC IN MINAS GERAIS / BRAZIL}

\begin{abstract}
COVID-19 pandemic has managers and researchers challenged in the search for better strategies to face diseases and prevent a collapse the health system. This article analyzes different strategies of social isolation and to verify its effects in reducing the number of infected and deaths caused by COVID-19. Through mathematical modeling and computer simulation, we analyze three different types of social isolation for the state of Minas Gerais/Brazil. We consider a rigid social isolation as $80 \%$ reduction rate in social contact, applied in uninterrupted intervals of 48 (forty-eight) days or distributed in interspersed with optimized social isolation, making possible provision of services and enable the provision of
\end{abstract}

income generation. The alternative that rigid social isolation was adopted only in the weekend, alternately with optimized isolation with a $55 \%$ reduction of social contact from Monday to Friday shows a reduction of $18.6 \%$ in total number of deaths and infections when compared with uninterrupted rigid social isolation for same time, reducing in $8.6 \%$ these cases when compare to scenario with actual average isolation rate maintained during the same period. These results allow the projection of a pandemic scenario in the state and assist in the analysis of alternatives for controlling of COVID-19 in Minas Gerais and Brazil.

KEYWORDS: mathematical model, coronavirus, quarantine, epidemic curve 


\section{INTRODUÇÃO}

O início do ano de 2020 foi marcado por um dos maiores desafios sanitários e epidemiológicos já conhecidos, fazendo a Organização Mundial da Saúde (OMS) declarar oficialmente o surto da doença COVID-19 como uma Emergência de Saúde Pública de Importância Internacional (ESPII). A doença causada pelo novo coronavírus (SARS-CoV-2) resulta no comprometimento do sistema respiratório (Síndrome Respiratória Aguda Grave -SARS) . Os primeiros casos foram relatados na cidade de Wuhan, na província de Hubei (China) em dezembro do ano anterior (OMS, 2020).

Em março, a OMS, em virtude da disseminação geográfica rápida que a COVID-19 tem apresentado, elevou o estado da contaminação à pandemia mundial, com 11.500 .302 casos confirmados no dia 07 de julho de 2020, afetando um total de 188 países (Johns Hopkins CSSE, 2020). Na América Latina, o Brasil lidera o número de casos notificados e mortes, tendo seu primeiro caso registrado no dia 26 de fevereiro de 2020, na cidade de São Paulo (Croda \& Garcia, 2020). Em Minas Gerais, com um registro de 78.643 infectados e 1.688 mortes até o dia 14/07/2020 (SES, 2020a), medidas como proibição de eventos, fechamento total de unidades de ensino e suspensão de comércios e serviços, transporte rodoviário, aquaviário e ferroviário e adoção do teletrabalho para servidores foram algumas das estratégias adotadas para o enfrentamento da doença (Aquino, Silveira, Pescarini, Aquino, \& Souza-Filho, 2020).

O combate à pandemia da COVID-19 tem sido manejado de diversas formas em cada país e estratégias diferentes são empregadas para conter o avanço da pandemia e achatar a curva epidêmica a fim de evitar a sobrecarga do sistema de saúde, variando de acordo com o contexto de cada local. $O$ isolamento e distanciamento social tem sido uma das estratégias mais abordadas, com variações na taxa de isolamento e período de duração. Seus efeitos têm sido estudados em diversos trabalhos(Geard et al., 2020; Kupferschmidt \& Cohen, 2020; Wilder-Smith \& Freedman, 2020) .

O impacto econômico do isolamento social tem sido uma preocupação constante, principalmente nos países que apresentam uma quantidade significativa de trabalhadores informais e com alto índice de desigualdade social, como é o caso do Brasil. Embora haja evidências de que o isolamento social a longo prazo não seja o fator determinante na piora da economia (Domingues et al., 2020). O fortalecimento do Sistema único de Saúde e de políticas de proteção social e apoio a populações em situação de vulnerabilidade são fundamentais para a aplicação de estratégias de isolamento eficazes e diferentes estratégias de isolamento podem ser alternativas eficazes para o enfrentamento da pandemia em Minas Gerais e no Brasil (Aquino et al., 2020; Castilho, Gondim, Marchesin, \& Sabeti, 2020; Lacerda \& Nepomuceno, 2020).

Com a rápida disseminação do vírus, a compreensão e previsão da dinâmica de propagação da doença, permite que as estratégias de controle e mitigação da pandemia sejam definidas de forma otimizada, possibilitando verificar a influência de cada uma destas na curva de transmissão. A adoção de modelos matemáticos para prever o comportamento de epidemias foi registrada pela primeira vez na última metade do século XVIII, com o estudo da dinâmica populacional da varíola 
(Caetano, 2010; Galante, 2008), e desde então é uma importante ferramenta no enfrentamento de doenças infecciosas e compreensão de epidemias (Codeço \& Coelho, 2012).

O modelo matemático possibilita uma melhor compreensão do comportamento dinâmico, este se mostra importante e eficaz para o estudo de doenças infecciosas, auxiliando na compreensão da onda endêmica e no entendimento da relação entre infectividade, recuperação e mortalidade. Estas informações podem inclusive auxiliar na compreensão do término da epidemia mesmo que ainda hajam suscetíveis na população, como ocorreu na epidemia causada pela H1N1 em 2009, no Brasil (Caetano, 2010; Codeço \& Coelho, 2012).

\section{METODOLOGIA}

\subsection{Contexto}

O presente trabalho foi desenvolvimento para análise da evolução da pandemia da COVID19, no estado de Minas Gerais/Brasil, com população estimada de 21.168.791 habitantes, distribuídos em 853 municípios (IBGE, 2019). O primeiro caso confirmado da doença no estado foi em 04 de março de 2020, sendo a primeira internação em decorrência da COVID-19 registrada no dia 16 de março e o primeiro óbito ocorrido no fim do mesmo mês (Secretaria Estadual de Saúde (SES), 2020d) .

A análise e validação do modelo foram realizadas com base nos dados fornecidos pela Secretaria Estadual de Saúde de MG (SES) (SES, 2020d), no período de 04/03/2020 a 31/12/2020. Considerando o início da pandemia no estado a data inicial dos dados fornecida pela SES, de acordo com os dados informados, o número inicial de infectados é igual a 6 . As taxas de isolamento foram obtidas a partir do monitoramento realizado pela InLoco ${ }^{1}$, empresa que trabalho com 0 monitoramento de dados de localização, e são apresentadas na Figura 1. No período considerado, a porcentagem de isolamento médio foi de $37,2 \%$.

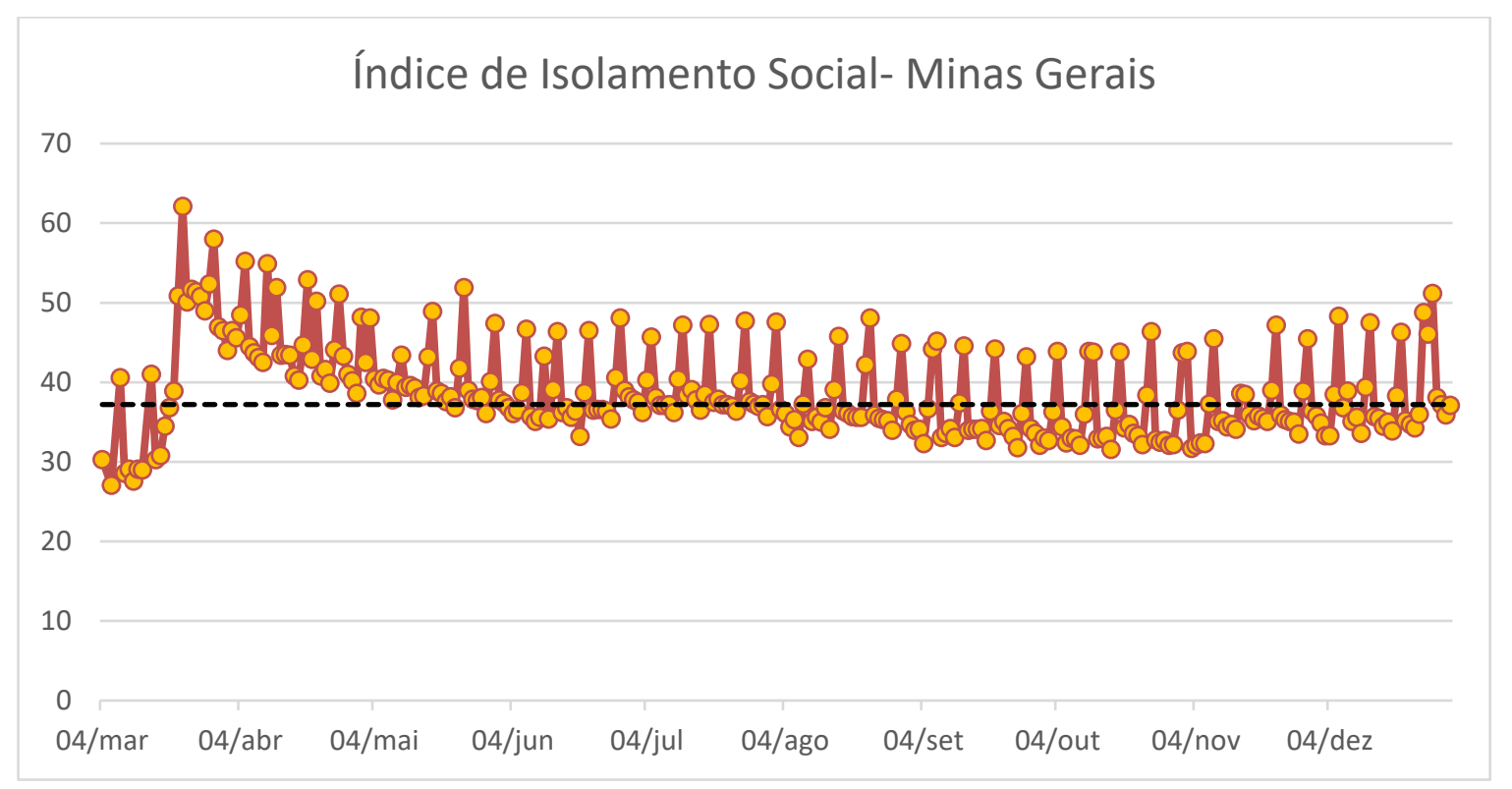

Figura 1: Índice de Isolamento Social de Minas Gerais. Fonte: InLoco 


\subsection{Modelagem Matemática}

Este trabalho tem natureza teórico-computacional e utilizou o software livre Scilab ${ }^{\circledR}$ (solucionador Isoda do pacote ODEPACK) para realização de simulações do modelo matemático para avaliação da dinâmica da COVID-19 no estado de Minas Gerais/Brasil. O modelo estudado é do tipo SEIR (Suscetível-Exposto-Infectado-Recuperado), considerando também a dinâmica de mortes devido a infecção do vírus. O modelo foi desenvolvido com base nos trabalhos de Eikenberry et al. (2020) e Jiwei et al. (2020) .

Para o seu desenvolvimento, considera-se a população em análise numerosa, o que permite ignorar efeitos aleatórios devido às diferenças de indivíduos e que a mesma é homogeneamente distribuída, o que faz com que infecciosos e susceptíveis estejam distribuídos aleatoriamente na área que a população vive. Além disso, considera-se que os infectados, uma vez recuperados, ficam imunes durante todo o período simulado e que tanto sintomáticos, assintomáticos e hospitalizados podem transmitir o vírus. O modelo obtido é apresentado nas Equações (1)-(8).

$$
\begin{aligned}
& \frac{d S}{d t}=-\beta(I+\eta A+l H)-p S+\lambda Q \\
& \frac{d Q}{d t}=p S-\lambda Q \\
& \frac{d E}{d t}=\beta(I+\eta A+l H)-\sigma E \\
& \frac{d I}{d t}=\alpha \sigma E-\varphi I-\gamma_{I} I \\
& \frac{d A}{d t}=(1-\alpha) \sigma E-\gamma_{A} A \\
& \frac{d H}{d t}=\varphi I-\delta H-\gamma_{H} H \\
& \frac{d R}{d t}=\gamma_{I} I+\gamma_{A} A+\gamma_{H} H \\
& \frac{d D}{d t}=\delta H
\end{aligned}
$$

na qual $S(t), Q(t), E(t), I(t), A(t), H(t), R(t) e D(t)$ são os indivíduos susceptíveis, em isolamento social, expostos, infectados sintomáticos, infectados assintomáticos, hospitalizados, recuperados e mortes, respectivamente. Define-se $N=S+E+I+A+R+H$ como a população total e $\beta$ como a taxa de transmissão por contato. Neste caso, foram desconsiderados nascimentos e efeitos migratórios. Considerou-se ainda que, ao início da pandemia, cada indivíduo infectado expõe outros 20 indivíduos ao vírus (Savi, Savi, \& Borges, 2020).

A escolha do modelo tipo SEIR em detrimento de um modelo mais simples do tipo SIR (Susceptível-Infectado-Recuperado) permite uma análise mais adequada do impacto do isolamento de indivíduos expostos na dinâmica de disseminação do vírus, embora o novo 
coronavírus tenha apresentado uma fase infectada não infecciosa (representada pelo indivíduo Exposto no modelo) muito rápida (Eikenberry et al., 2020; Jiwei et al., 2020).

Além disso, para o desenvolvimento do modelo, adotou-se como hipótese que pessoas em isolamento não estão em contato com o restante da população, não contribuindo com a taxa de transmissão (e consequentemente, não são contabilizadas em $\mathrm{N}$ ). As mortes consideradas são apenas de pessoas hospitalizadas, considerando o baixo número de testes realizados relacionados a mortes de indivíduos não-hospitalizados (Fiúza, 2020).

Uma vez que está sendo adotado o distanciamento social voluntário e mandatório em algumas regiões do estado, considerou-se que $\beta=\beta(t)$, conforme definido na Equação (9a).

$$
\beta(t)= \begin{cases}\beta_{0}, & t<t_{0} \\ \beta_{\text {min }}+\left(\beta_{0}-\beta_{\text {min }}\right) e^{-r\left(t-t_{0}\right)}, & t \geq t_{0}\end{cases}
$$

na qual $\beta_{\min }$ é a taxa de transmissão por contato mínima e $r$ é a taxa de decréscimo da taxa de contato.

A definição de $\beta(t)$ como uma função do tempo, definida de forma diferente para momentos distintos da pandemia, foi feita de acordo com o comportamento observados nos dados reais divulgados pela Secretaria de Saúde do Estado de Minas Gerais. Observa-se nos dados reais que após $t \approx 4 t_{0}$, há um aumento na taxa de transmissão, que se aproxima do valor definido no início da pandemia. Assim, o cenário inicial, utilizado para validação do modelo, é reproduzido de forma análoga posteriormente para os mesmos valores de intervalos de tempo e $\beta(t)$ a fim de simular uma projeção da pandemia. Para a simulação da projeção, $\beta(t)$ é definido pela Equação (9b).

$$
\beta(t)=\left\{\begin{array}{cc}
\beta_{0}, & t \leq t_{0} \\
\beta_{\text {min }}+\left(\beta_{0}-\beta_{\text {min }}\right) e^{-r\left(t-t_{0}\right),}, \quad t_{0}<t \leq 4 t_{0} \\
\beta_{0}, \quad 4 t_{0}<t \leq 5 t_{0} \\
\beta_{\text {min }}+\left(\beta_{0}-\beta_{\text {min }}\right) e^{-r\left(t-t_{0}\right),} \quad 5 t_{0}<t
\end{array}\right.
$$

Os demais parâmetros e os valores adotados bem como as condições iniciais para a simulação são apresentados na Tabela 1.

$O$ isolamento é descrito através do balanceamento entre as pessoas que entram e saem da categoria isolado, descrita pelos parâmetros p e $\lambda$ (Castilho et al., 2020). O valor definido para a taxa de liberação da quarentena teve como base os estudos de Jiwei et al. (2020) e Castilho et al. (2020), cujos modelos foram aplicados para China e São Paulo, respectivamente, e a partir do ajuste do modelo para os dados coletados para Minas Gerais. 
Tabela 1: Parâmetros do Modelo e Condições Iniciais

\begin{tabular}{|c|c|c|c|}
\hline Parâmetro & Descrição & Valor & Referência \\
\hline $\boldsymbol{\beta}_{\min }$ & $\begin{array}{l}\text { Taxa de transmissão por contato } \\
\text { mínima }\end{array}$ & $0,51 \mathrm{dia}^{-1}$ & Eikenberry et al. (2020) \\
\hline $\boldsymbol{\beta}_{\mathbf{0}}$ & Taxa de transmissão por contato inicial & $1,45 \mathrm{dia}^{-1}$ & Eikenberry et al. (2020) \\
\hline $\boldsymbol{r}$ & $\begin{array}{l}\text { Taxa de decréscimo da taxa de contato } \\
\text { Tempo a partir do qual há um }\end{array}$ & $0,01 \mathrm{dia}^{-1}$ & Assumido \\
\hline$t_{0}$ & $\begin{array}{l}\text { Tempo a partir do qual há um } \\
\text { decréscimo na taxa de transmissão } \\
\text { Coeficiente de transmissão relativa }\end{array}$ & 65 dias & Assumido \\
\hline $\boldsymbol{\eta}$ & $\begin{array}{l}\text { para assintomáticos em relação aos } \\
\text { sintomáticos }\end{array}$ & 0,03 & Assumido \\
\hline $\boldsymbol{l}$ & $\begin{array}{l}\text { Coeficiente de transmissão relativa } \\
\text { para hospitalizados em relação aos } \\
\text { sintomáticos }\end{array}$ & 0,75 & Assumido \\
\hline $\boldsymbol{\sigma}$ & $\begin{array}{l}\text { Tempo de transição de exposto para } \\
\text { infeccioso }\end{array}$ & $1 / 7 \mathrm{dia}^{-1}$ & OMS (2020) \\
\hline$\alpha$ & $\begin{array}{l}\text { Fração de infecções que se tornam } \\
\text { sintomáticas }\end{array}$ & 0,1429 & Eikenberry et al. (2020) \\
\hline$\varphi$ & Taxa de hospitalização do sintomáticos & $0,115 \mathrm{dia}^{-1}$ & SES (2020d) \\
\hline$\gamma_{I}$ & Taxa de recuperação dos sintomáticos & $1 / 10 \mathrm{dia}^{-1}$ & Eikenberry et al. (2020) \\
\hline$\gamma_{A}$ & $\begin{array}{l}\text { Taxa de recuperação dos } \\
\text { assintomáticos }\end{array}$ & $1 / 7 d i a^{-1}$ & Eikenberry et al. (2020) \\
\hline$\gamma_{H}$ & $\begin{array}{l}\text { Taxa de recuperação dos } \\
\text { hospitalizados }\end{array}$ & $1 / 14 \mathrm{dia}^{-1}$ & Eikenberry et al. (2020) \\
\hline $\boldsymbol{\delta}$ & Taxa de mortalidade & $0,07 \mathrm{dia}^{-1}$ & Eikenberry et al. (2020) \\
\hline$\lambda$ & Taxa de liberação da quarentena & & $\begin{array}{l}\text { Castilho et al.(2020); Jia } \\
\text { et al. (2020) }\end{array}$ \\
\hline \multicolumn{4}{|c|}{ Condições iniciais } \\
\hline$N(\mathbf{0})$ & População & 14754647 & IBGE (2019) \\
\hline$S(0)$ & Indivíduos susceptíveis & 14754521 & Eikenberry et al. (2020) \\
\hline$E(\mathbf{0})$ & Indivíduos expostos & 120 & Savi et al. (2020) \\
\hline$I(0)$ & Indivíduos infectados sintomáticos & 6 & $\begin{array}{l}\text { (Secretaria Estadual de } \\
\text { Saúde (SES), 2020d) }\end{array}$ \\
\hline$A(\mathbf{0})$ & Indivíduos infectados assintomáticos & 0 & Assumido \\
\hline $\boldsymbol{H}(\mathbf{0})$ & Indivíduos hospitalizados & 0 & Assumido \\
\hline$Q(\mathbf{0})$ & Indivíduos isolados & 4470658 & $\begin{array}{l}\text { InLoco(2020); IBGE } \\
\text { (2019) }\end{array}$ \\
\hline
\end{tabular}

A taxa de entrada do isolamento é a relação entre a porcentagem de indivíduos que de fato se isolam voluntariamente $(\xi)$ e o tempo médio gasto para entrar em isolamento $\left(\gamma_{q}\right)$, expressa na Equação (10). 


$$
p=\frac{\xi}{\gamma_{q}}
$$

Assume-se, que $p=0$ indica nenhum isolamento. $O$ valor adotado para $\gamma_{q}=1 / 2$ indica que são necessários 2 (dois) dias para atingir tal porcentagem de isolamento. Este foi definido com base no período de publicação dos decretos no Estado de Minas Gerais, que em sua maioria são publicados na sexta, passando a ter validade na segunda-feira (Secretaria do Estado da Fazenda de Minas Gerais (SEF), 2020). Este parâmetro foi mantido constante, independente da porcentagem de indivíduos em isolamento.

As projeções do número de casos da COVID-19 e da quantidade de mortes são baseadas em três estratégias de isolamento social distintas, definidas hipoteticamente de acordo com algumas referências da literatura, de modo que todas possuíssem um período de 48 (quarenta e oito) dias de lockdown, definido como $80 \%$ de isolamento conforme o Lacerda e Nepomuceno ( 2020).

Cada estratégia foi implementada a partir do dia 01/08/2020, data inicial definida de modo que a estratégia que necessitasse de um período maior para ser implementada, fosse finalizada ao fim do ano de 2020, a fim de verificar os impactos de cada estratégia com os dados reais obtidos. Adicionalmente, levando em consideração a possível adaptação que a população deverá realizar para conseguir efetivamente alcançar o lockdown, considerou-se um período de transição de 10 dias ( 22 a 31/07/2020) com 55\% de isolamento social. O detalhamento das estratégias estudadas está disposto a seguir:

- Estratégia de isolamento 1: 48 (quarenta e oito) dias ininterruptos de lockdown.

- Estratégia de isolamento 2: Lockdown apenas aos sábados e domingos, totalizando 48 (quarenta e oito) dias, intercalados com 5(cinco) dias de isolamento otimizado (55\%) de segunda a sexta.

- Estratégia de isolamento 3: Lockdown por 24 (vinte e quatro) dias ininterruptos, intercalados com 15(quinze) dias de isolamento otimizado (55\%) e novamente, 24 (vinte e quatro) dias de lockdown.

Após cada estratégia, a taxa de isolamento média obtida no período de 04/03 a $14 / 07 / 2020$, igual a 39,92\%, é mantida até o fim do período de simulação. É importante enfatizar que os feriados nacionais $(07 / 09,12 / 10,02 / 11$ e 25/12) também foram considerados na contagem dos 48 dias a fim de otimizar o isolamento. Caso a estratégia não permaneça até tal data, considerou-se que o estado poderia adiantar o recesso do feriado como já tem sido adotado em outros locais.

\section{RESULTADOS E DISCUSSÃO}

O modelo foi ajustado aos dados reais do período de 04/03 a 31/12/2020, apresentados pela Secretaria Estadual de Saúde de Minas Gerais (SES). É importante ressaltar que a partir do mês de outubro, a SES passou a não informar os dados diários aos domingos e feriados. Neste caso, 
os autores optaram por não considerar mortes e infectados neste dia, o que afeta o perfil diário, porém, não influencia o total de mortes ou infectados obtidos. Após o ajuste, foi realizada uma projeção até o dia 24/08/2021, totalizando 540 dias de análise a partir do primeiro caso confirmado, conforme o boletim emitido pelo SES (2020d). Os resultados são apresentados na Figura 2.

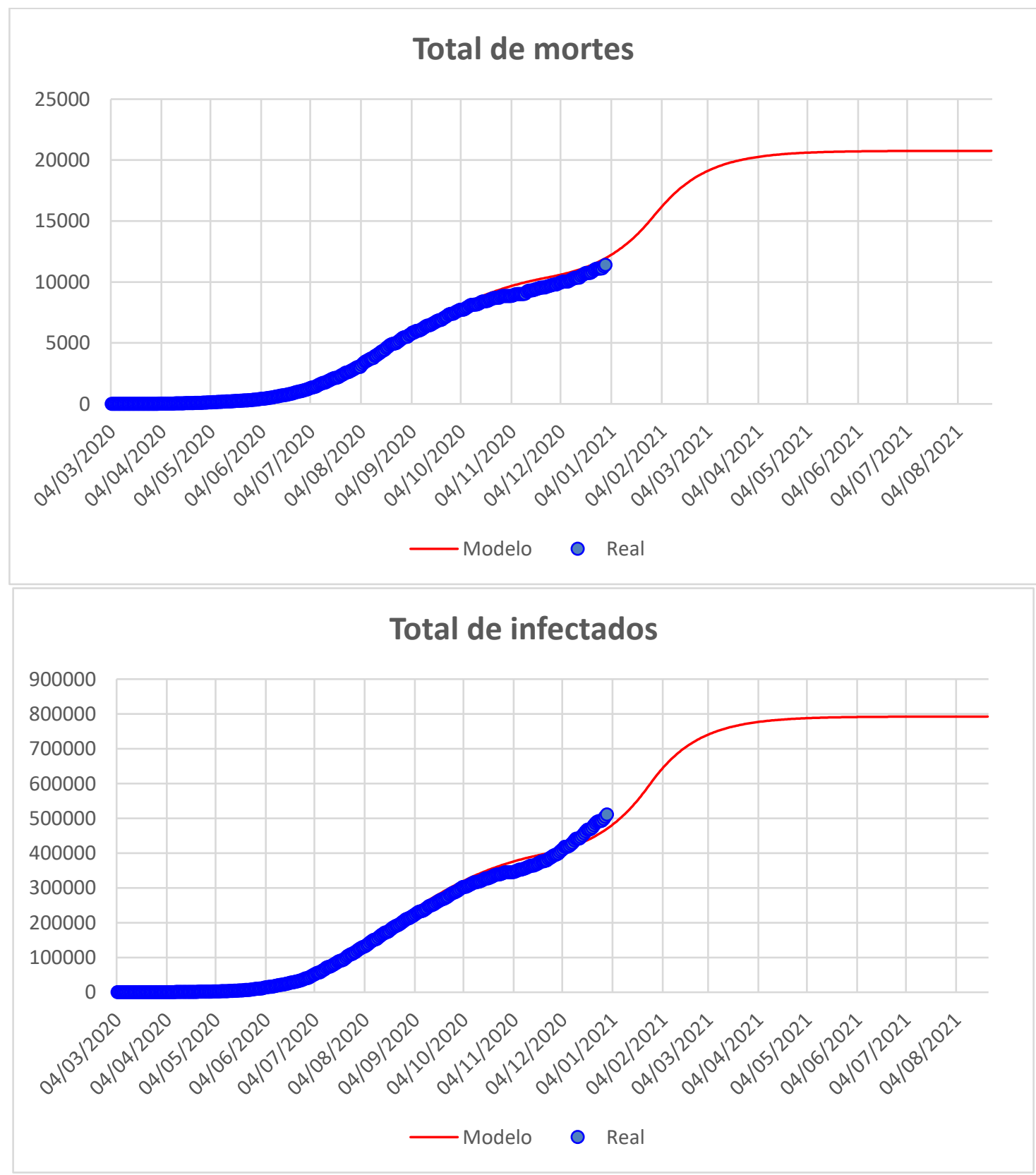

Figura 2: Total de mortes e infectados provenientes da COVID-19 em Minas Gerais no período de 04/03/2020 a $24 / 08 / 2021$ 
Pelos resultados apresentados, é possível verificar que o modelo tem um ajuste adequado aos dados reais, com coeficiente de ajuste da curva de $\mathrm{R}^{2}=0,9913$ em relação ao número total de mortos e de $\mathrm{R}^{2}=0,9912$ em relação ao número total de infectados no período analisado.

Caso a previsão realizada pelo modelo se confirme, mantendo-se a taxa média de isolamento nos meses seguintes também em torno de $37,2 \%$, estima-se, num período de 540 dias, 20.761 mortos no período e um total de 792.451 infectados, caracterizando uma taxa de letalidade de 2,62\%. O perfil de distribuição diário de mortes e infectados é apresentado na Figura 3.

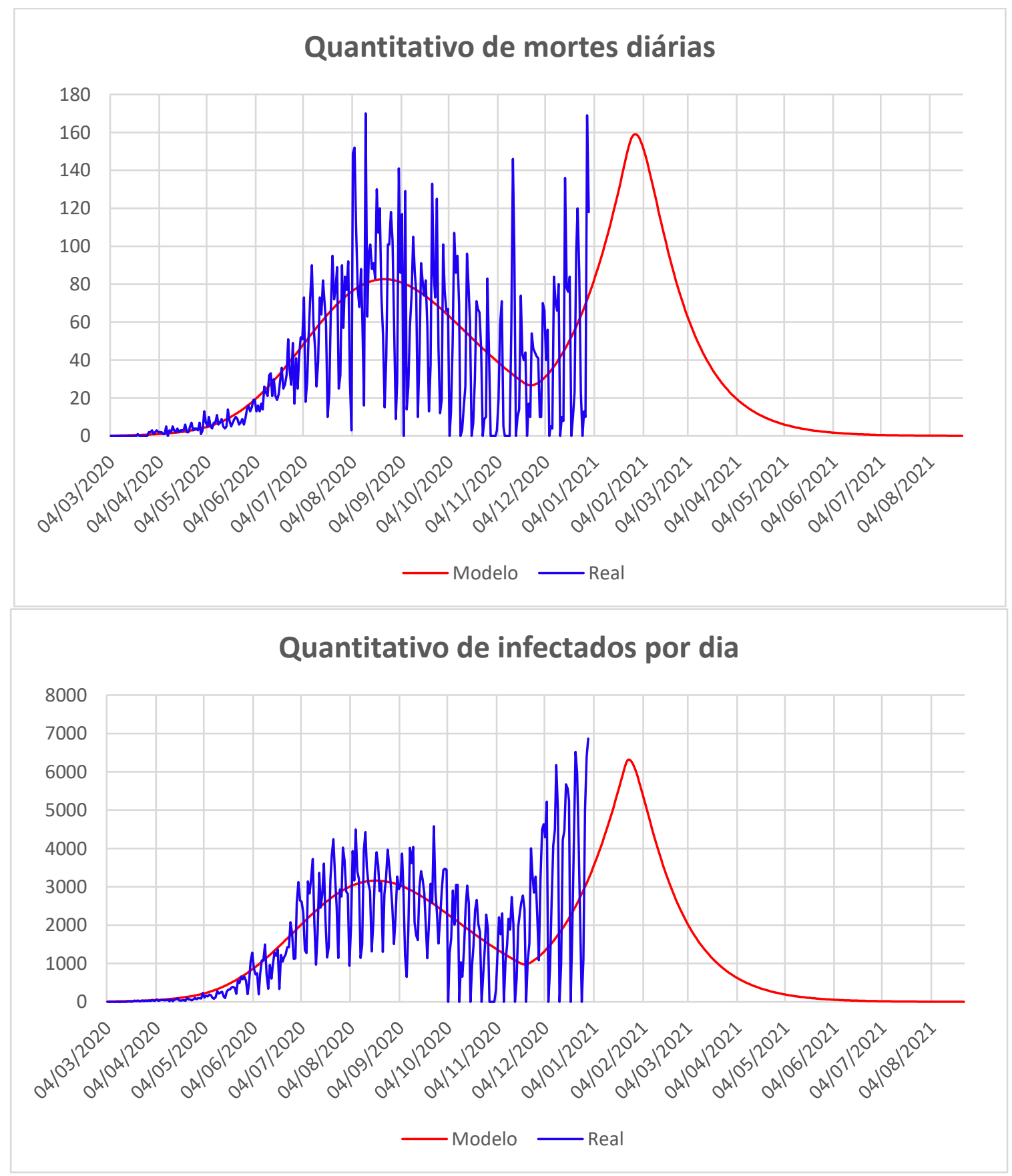

Figura 3: Perfil de distribuição diária do número de mortos e infectados pela COVID-19 no estado de Minas Gerais

Segundo o modelo, observa-se o primeiro pico de novos casos diários por volta do dia 22/08/2020, totalizando, aproximadamente, 3160 casos/dia e um total de 83 mortes diárias. De HOLOS, Ano 36, v.5, e11460, 2020 
acordo com as projeções apresentadas pelo Epidemiológico e Assistencial (Edição Especial) no 11 (Secretaria Estadual de Saúde (SES), 2020c), disponibilizado pelo SES, estima-se que estes parâmetros são 2000 casos/dia e 51 mortes diárias, com o pico da curva definido para o dia $18 / 07 / 2020$, indicando um aumento de $7,65 \%$ do número de casos e mortes diárias da previsão do modelo em relação a estimativa do SES.

Esta diferença se deve, principalmente, ao fato de a estimativa apresentada basear-se nos dados até o dia 31/12/2020, enquanto o relatório apresentado pela SES tem como base os dados até o dia 24/06/2020. Observa-se que a curva de distribuição obtida pelo modelo segue a tendência dos dados reais, com uma discrepância principalmente com os dados apresentados a partir do 4ㅇ mês (julho). Neste mesmo período, houve um aumento considerável na média de testagem diária realizada, conforme o boletim Epidemiológico e Assistencial (Edição Especial) no 12 (Secretaria Estadual de Saúde (SES), 2020b). Ressalta-se ainda que o número de confirmados diários não corresponde ao número de casos ocorridos nas últimas $24 \mathrm{~h}$, o que pode resultar em uma divergência das predições diárias do modelo em relação aos dados reais, porém, sem grande influência na eficácia do modelo quando se analisa os dados acumulados. Assim, através da análise dos dados reais, nota-se que o primeiro pico ocorreu entre o dia 12 e 25/08/2020.

Observa-se ainda, caso o cenário se repita, um novo pico no fim do mês de janeiro de 2021 pode ser observado com um total de, aproximadamente, 159 mortes por dia, o que representa um aumento de $82,76 \%$ em relação ao primeiro pico estimado. Quando se considera o número de casos diários, esse aumento seria de $99,78 \%$, comportamento este em concordância com os principais estudos que indicam uma segunda onda mais intensa que a primeira na pandemia do novo coronavírus, reforçando a necessidade de intensificar as medidas preventivas como o isolamento.

Após o ajuste do modelo aos dados reais, as estratégias de isolamento apresentadas na Seção 2 foram implementadas a fim de verificar qual delas seria mais efetiva na redução do número de casos e mortos diários e acumulados. As taxas de isolamento consideradas para cada estratégia são apresentadas na Figura 4. 


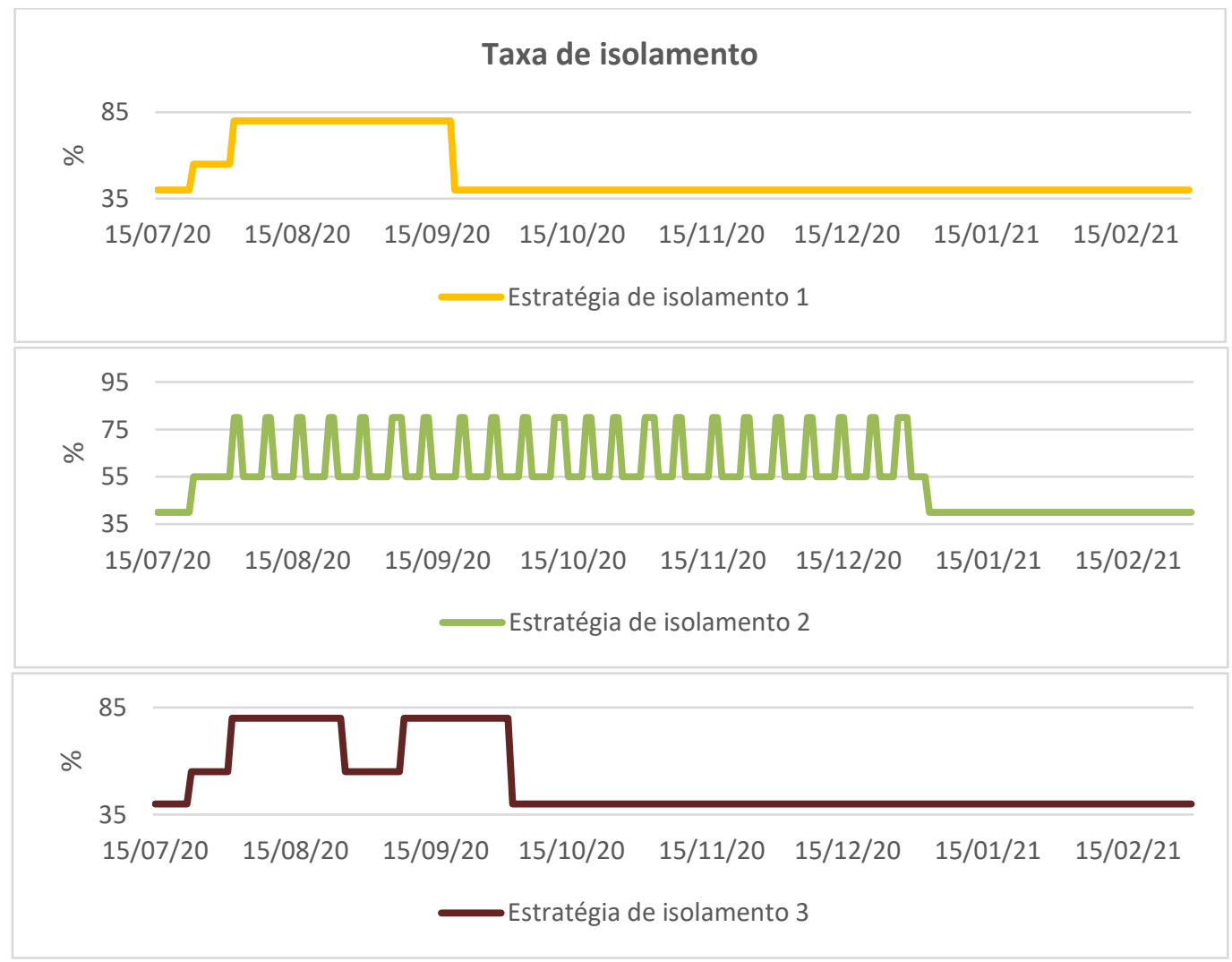

Figura 4: Estratégias de isolamento

Os efeitos das estratégias apresentadas no número total de infectados e mortes são apresentados na Figura 5. Os resultados são apresentados juntamente com a manutenção da taxa média de isolamento 


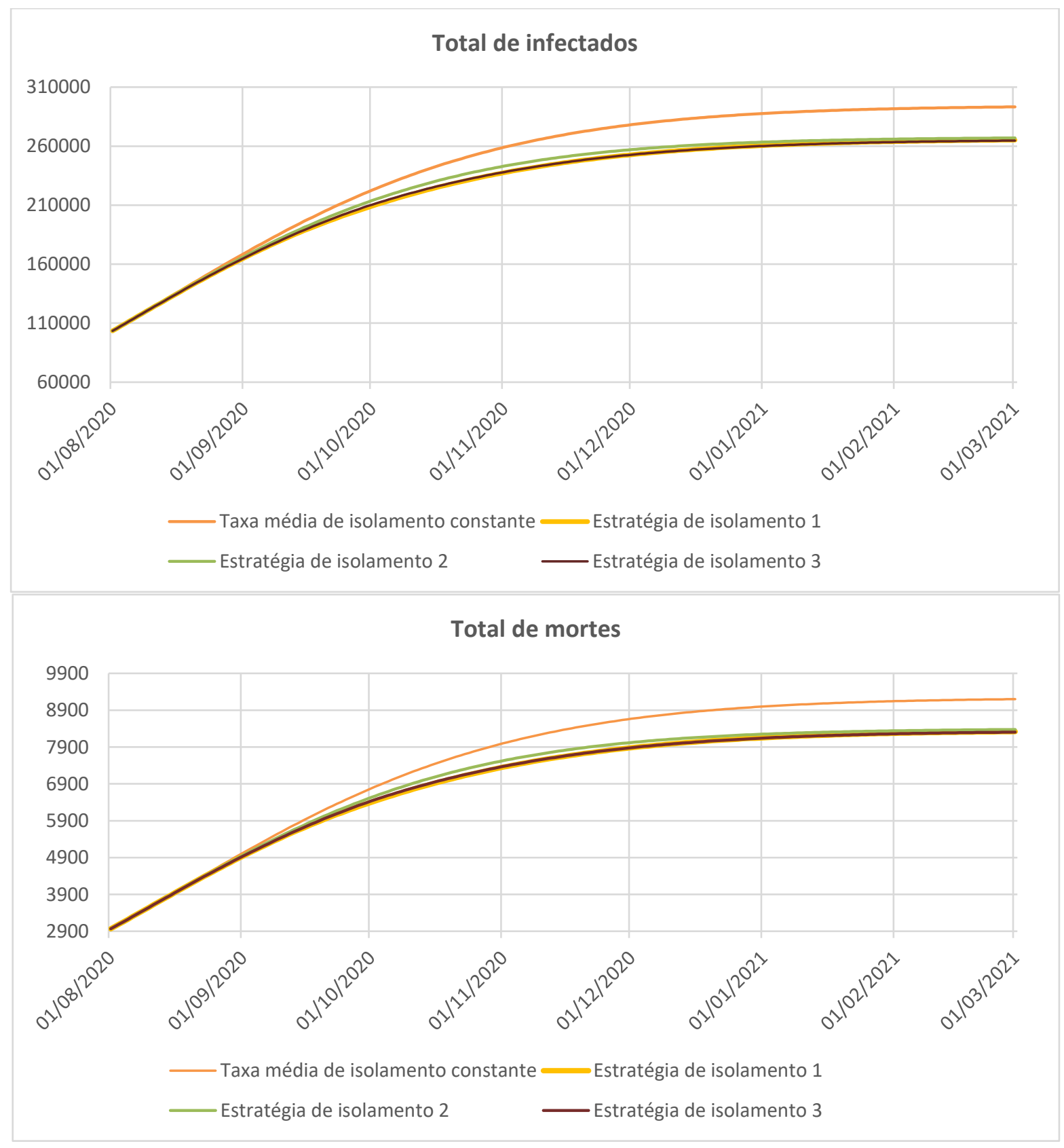

Figura 5:Total de infectados e mortes para as diferentes estratégias de isolamento

Pelos resultados apresentados, é possível verificar que as três estratégias simuladas conseguem reduzir o número total de mortes e infectados no período apresentado de modo significativo quando comparado com a manutenção do isolamento na taxa constante média apresentada anteriormente. A Tabela 2 apresenta o número total de mortos e infectados no período simulado para as quatro abordagens apresentadas:

Tabela 2:Total de mortos e infectados para as estratégias de isolamento avaliadas

\begin{tabular}{c|c|c}
\hline Isolamento & Mortes & Infectados \\
\hline $\begin{array}{c}\text { Isolamento com taxa média } \\
\text { constante }\end{array}$ & 9.201 & 293.254 \\
Estratégia de isolamento 1 & 8.557 & 272.741
\end{tabular}




\section{Estratégia de isolamento 2 \\ Estratégia de isolamento 3

As Estratégias de isolamento 1,2,3 alcançaram uma redução no número de mortes de 7\% (644 mortes), 8,3\% (764 mortes) e 7,6\% (702 mortes) no período comparado com o isolamento com taxa média constante. Dentre as opções apresentadas, a Estratégia de isolamento 2 foi a que alcançou um maior percentual de redução, resultado que assemelha-se ao encontrado por Lacerda e Nepomuceno (2020).

Ao analisar o número total de infectados o impacto é ainda mais significativo e a Estratégia de isolamento 2, novamente é a que apresenta efeitos mais significativos, reduzindo o número total de infectados em 24.371 indivíduos. As outras alternativas (1 e 3) também apresentam uma redução de aproximadamente $7 \%$ (20.513 casos) e 7,6\% (22.367 casos) em relação ao isolamento constate. O perfil diário de mortes e infectados obtido para cada uma das estratégias é apresentado na Figura 6.

Observa-se que as três propostas apresentam perfis semelhantes e uma redução no pico apresentado, quando comparado ao isolamento com taxa constante igual 39,95\%. As Estratégia de Isolamento 1 e 3 apresentam perfis muito semelhantes e pico igual a 65 mortes diárias e 2.097 infectados/dia, enquanto o isolamento com taxa constante atinge um pico de 67 mortes diárias e 2.157 infectados diariamente. Já a Estratégia de Isolamento 2 alcança o máximo de 66 mortes diárias e 2.103 infectados.

O percentual de redução de cada estratégia abordada em relação à manutenção da taxa de isolamento média por todo o período considerado é apresentado na Tabela 3.

Tabela 3:Taxa de redução obtidas pelas estratégias propostas

\begin{tabular}{c|c|c}
\hline Estratégia & $\begin{array}{c}\text { Redução de mortes diárias } \\
(\%)\end{array}$ & $\begin{array}{c}\text { Redução de infectados } \\
\text { diários (\%) }\end{array}$ \\
\hline Estratégia de Isolamento 1 & 2,99 & 2,78 \\
Estratégia de Isolamento 2 & 1,49 & 2,5 \\
Estratégia de Isolamento 3 & 2,99 & 2,78 \\
\hline
\end{tabular}

É importante ressaltar que a adoção do lockdown ininterrupto por 30 dias obteve efeitos semelhantes à Estratégia de Isolamento 3 (dois períodos de lockdown de 15 dias intercalados com outros 15 dias de isolamento otimizado) quando se analisa o perfil diários porém, esta última obteve melhores resultados quando analisa-se os perfis totais de mortes e infectados, apresentando um percentual de redução para esses perfis de $9 \%$ em relação ao resultado obtido adotando-se a Estratégia de Isolamento 1. 


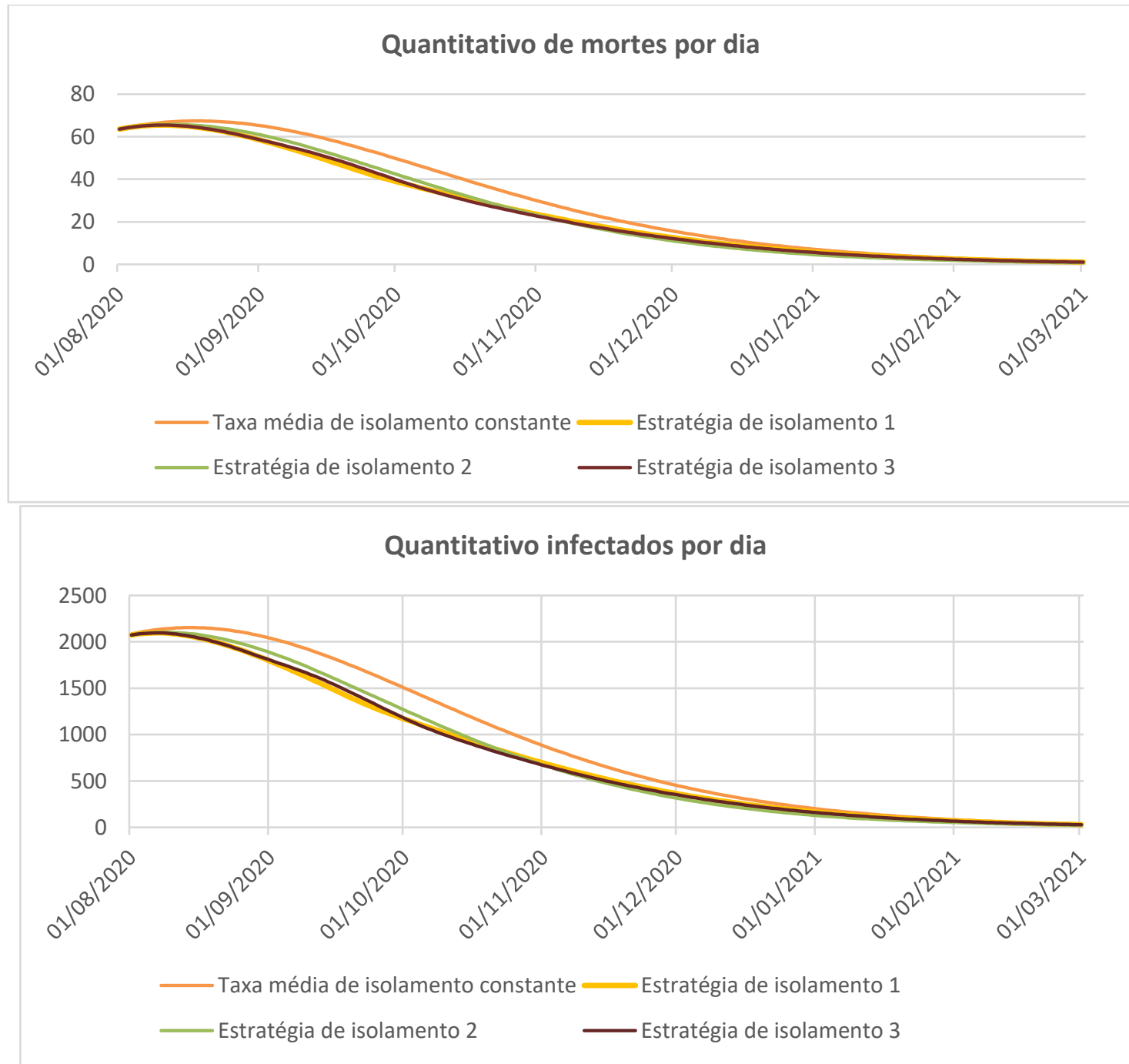

Figura 6: Perfil diário de mortes e infectados para as estratégias de isolamento

A adoção da Estratégia de isolamento 2 obteve os melhores resultados para os números acumulados e apresenta uma maior flexibilidade, o que facilitaria sua extensão por um período maior, minimizando os efeitos na economia. Esta estratégia apresenta um percentual de redução em relação às Estratégias de isolamento 1 e 3 de, aproximadamente, $18,6 \%$ e 8,8\%, respectivamente.

Além disso, embora as reduções nos perfis diários pareçam mínimas em valores absolutos, percentualmente as Estratégias de Isolamento 1, 2 e 3 apresentam uma redução significativa. Ressalta-se ainda que as estratégias foram limitadas a 30 dias, porém ao estendê-las por período maior, o resultado absoluto total e diário poderia ser ainda mais significativo.

Além disso, os resultados obtidos para cada uma das estratégias em relação aos dados reais para o dia 31/12/2020, quando a taxa de isolamento média é $37,2 \%$. Os resultados são apresentados na Tabela 4. 
Tabela 4-Total de mortos e infectados reais e para as estratégias de isolamento avaliadas até o dia 31/12/2020

\begin{tabular}{c|c|c}
\hline Isolamento & Mortes & Infectados \\
\hline Dados reais & 11.403 & 511.430 \\
Estratégia de isolamento 1 & 8.147 & 260.331 \\
Estratégia de isolamento 2 & 8.246 & 263.313 \\
Estratégia de isolamento 3 & 8.139 & 259.710 \\
\hline
\end{tabular}

Observa-se que a adoção de estratégias de isolamento reduz de forma significativa os números totais de mortes e infectados. A Estratégia de Isolamento 1 apresenta uma redução de $28,55 \%$ em relação ao número de mortes e $49,1 \%$ quando analisa-se o número total de infectados. Analogamente para as Estratégias de Isolamento 2 e 3, as reduções para infectados são 48,5\% e $49,21 \%$, respectivamente, e para o número de mortes, $27,69 \%$ e $28,62 \%$. Tais resultados reforçam a eficácia de medidas de isolamento adequadas e mais rígidas, mesmo que por curtos períodos de tempo, no combate ao coronavírus.

\section{CONCLUSÕES}

O isolamento social é uma importante estratégia para o enfrentamento da pandemia da COVID-19 no mundo. A definição da duração e da taxa de isolamento a partir do contexto social e econômico de cada local é uma tarefa complexa e a avaliação de diferentes estratégias permitem a otimização das escolhas adequadas para cada localidade.

O modelo desenvolvido para representar a evolução da pandemia do coronavírus em Minas Gerais apresentou um resultado satisfatório, com ajuste adequado aos dados reais apresentados pela Secretaria Estadual de Saúde do estado, podendo ser considerado como uma ferramenta útil para a realização de projeções da evolução da pandemia e direcionamento na tomada de decisões de ações para o combate ao coronavírus. É importante ressaltar que neste modelo não foram considerados casos de reinfecção, embora já se tenha conhecimento de algumas ocorrências, uma vez que os casos relatados ainda são poucos e diversos estudos tem sido realizados para verificar as possibilidades ou circunstâncias para a reinfecção (Bao et al., 2020; Feng et al., 2020).

A adoção de diferentes períodos de isolamento social rígido (lockdown) com alternância de períodos de maior flexibilidade mostrou-se uma alternativa eficaz na diminuição do número total de mortes e infectados no estado de Minas Gerais, alcançando taxas de até 8,3\% de redução em relação à alternativa de manter o isolamento constante na taxa média registrada no período de 04/03/2020 a 14/07/2020.

Dentre as estratégias analisadas, a Estratégia de Isolamento 2 apresenta melhores resultados quando analisado o número total de mortes e infectados no período, porém, as taxas de redução do quantitativo diário de casos e mortes são maiores quando se adota períodos maiores de lockdown consecutivos, como nas Estratégias de Isolamento 1 e 3 . Enfatiza-se aqui que, 
embora as duas estratégias adotem períodos diferentes de isolamento rígido, não houve diferença nas respostas obtidas ao adotar-se o lockdown por 30 dias consecutivos ou 15 dias alternados com igual período de isolamento otimizado.

Embora este trabalho não tenha analisado fatores econômicos, a definição de estratégias alternativas possibilitaria a flexibilização, aumentando oportunidades para a oferta de serviços e geração de renda. Entretanto, é evidente que o isolamento realizado hoje em média no estado ainda não é a taxa adequada para evitar a sobrecarga no sistema de saúde e garantir uma redução significativa nos números de casos. Porém, é importante ressaltar que o isolamento aplicado de maneira isolada, sem o fortalecimento do Sistema Único de Saúde e adoção de medidas protetivas para pessoas em vulnerabilidade social, além de fortalecimento de outras medidas não medicamentosas com a higienização adequada e o uso de máscaras, não será eficaz no enfrentamento da COVID-19 no Brasil e em Minas Gerais.

Ainda assim, é evidente a eficácia da adoção do isolamento social para o achatamento da curva epidêmica da COVID-19 em Minas Gerais e que a definição de estratégias adequadas de isolamento possibilita um equilíbrio entre aspectos sociais e econômicos, favorece os diversos setores e garante a eficácia no enfrentamento e minimização dos efeitos da pandemia na sociedade.

\section{REFERÊNCIAS}

Aquino, E. M. L., Silveira, I. H., Pescarini, J. M., Aquino, R., \& Souza-Filho, J. A. de. (2020). Medidas de distanciamento social no controle da pandemia de COVID-19: potenciais impactos e desafios no Brasil. Ciência \& Saúde Coletiva, 25, 2423-2446.

Bao, L., Deng, W., Gao, H., Xiao, C., Liu, J., Xue, J., ... Qin, C. (2020). Lack of Reinfection in Rhesus Macaques Infected with SARS-CoV-2. bioRxiv, 2020.03.13.990226. https://doi.org/10.1101/2020.03.13.990226

Caetano, M. T. P. (2010). Modelagem matemática da Influenza A (H1N1). Universidade Estadual de Campinas (UNICAMP), Campinas.

Castilho, C., Gondim, J. A. M., Marchesin, M., \& Sabeti, M. (2020). Assessing the efficiency of different control strategies for the coronavirus (COVID-19) epidemic. arXiv preprint arXiv:2004.03539.

Codeço, C. T., \& Coelho, F. C. (2012). Modelagem de doenças transmissíveis. Oecologia Australis, 16(1), 110-116.

Croda, J. H. R., \& Garcia, L. P. (2020). Resposta imediata da Vigilância em Saúde à epidemia da COVID-19. Epidemiologia e Serviços de Saúde, 29(1-3).

Domingues, E., Guedes, G., Ribeiro, R., Magalhães, A., Freire, D., Santos, R., ... Miyajima, D. (2020). Cenários de isolamento social da COVID19 e impactos econômicos em Minas Gerais.

Eikenberry, S. E., Mancuso, M., Iboi, E., Phan, T., Eikenberry, K., Kuang, Y., ... Gumel, A. B. (2020). 
To mask or not to mask: Modeling the potential for face mask use by the general public to curtail the COVID-19 pandemic. Infectious Disease Modelling.

Feng, Z., Diao, B., Wang, R., Wang, G., Wang, C., Tan, Y., ... Chen, Y. (2020). The Novel Severe Acute Respiratory Syndrome Coronavirus 2 (SARS-CoV-2) Directly Decimates Human Spleens and $\begin{array}{lll}\text { Lymph Nodes. medRxiv, 2020.03.27.20045427. } & \text { 20 }\end{array}$ https://doi.org/10.1101/2020.03.27.20045427

Fiúza, P. (2020). 70\% das mortes por Síndrome Respiratória em Minas são por causa indeterminada e governo admite falha em testes. Recuperado 11 de julho de 2020, de G1 website: https://g1.globo.com/mg/minas-gerais/noticia/2020/06/24/70percent-das-mortespor-sindrome-respiratoria-em-minas-sao-por-causa-indeterminada-e-governo-admite-falhaem-testes.ghtml

Galante, G. (2008). Epidemiologia matemática e computacional. Artigo Científico, Unioeste.

Geard, N., Giesecke, J. A., Madden, J. R., McBryde, E. S., Moss, R., \& Tran, N. H. (2020). Modelling the economic impacts of epidemics in developing countries under alternative intervention strategies. In Environmental Economics and Computable General Equilibrium Analysis (p. 193214). Springer.

InLoco. (2020). Mapa Brasileiro da COVID-19. Recuperado 13 de julho de 2012, de https://mapabrasileirodacovid.inloco.com.br/pt/

Instituto Brasileiro de Geografia e Estatística (IBGE). (2019). População estimada.

Jia, J., Ding, J., Liu, S., Liao, G., Li, J., Duan, B., ... Zhang, R. (2020). Modeling the control of COVID19: Impact of policy interventions and meteorological factors. arXiv preprint arXiv:2003.02985.

Johns Hopkins CSSE. (2020). COVID-19 Dashboard by the Center for Systems Science and Engineering (CSSE) at Johns Hopkins University (JHU). Recuperado 9 de julho de 2020, de ArcGIS

website: https://www.arcgis.com/apps/opsdashboard/index.html\#/bda7594740fd40299423467b48e 9ecf6

Kupferschmidt, K., \& Cohen, J. (2020). Can China's COVID-19 strategy work elsewhere? American Association for the Advancement of Science.

Lacerda, M. J., \& Nepomuceno, E. G. (2020). Uma nova estratégia de controle para o lockdown na pandemia da COVID-19.

Organização Mundial da Saúde (OMS). (2020). Folha informativa - COVID-19 (doença causada pelo novo Coronavírus. Recuperado 9 de julho de 2020, de OPAS: Brasil website: https://www.paho.org/bra/index.php?option=com_content\&view=article\&id=6101:covid19 \&ltemid $=8 \% 0 A 75$

Savi, P. V., Savi, M. A., \& Borges, B. (2020). A Mathematical Description of the Dynamics of 
Coronavirus Disease (COVID-19): A Case Study of Brazil. Recuperado de http://arxiv.org/abs/2004.03495

Secretaria do Estado da Fazenda de Minas Gerais (SEF). (2020). Coronavírus- Decretos e Resoluções. Recuperado 14 de julho de 2020, de http://www.fazenda.mg.gov.br/coronavirus/instrumentos-normativos/decretos-eresolucoes/

Secretaria Estadual de Saúde (SES). (2020a). Boletim Epidemiológico. Recuperado de http://coronavirus.saude.mg.gov.br/boletim

Secretaria Estadual de Saúde (SES). (2020b). Boletim Epidemiológico e Assistencial (Edição Especial) $n^{\circ}$ 12. Recuperado de http://coronavirus.saude.mg.gov.br/images/boletim/07julho/01-07_Boletim_Edicao-Especial-N11.pdf

Secretaria Estadual de Saúde (SES). (2020c). Boletim Epidemiológico e Assistencial COVID-19 (Edição $\quad$ Especial) $\quad n^{\circ} \quad 11 . \quad$ Recuperado de http://coronavirus.saude.mg.gov.br/images/boletim/07-julho/08-07Boletim_Edicao_Especial_N12.pdf

Secretaria Estadual de Saúde (SES). (2020d). Painel de Monitoramento dos Casos. Recuperado 3 de julho de 2020, de http://coronavirus.saude.mg.gov.br/painel

Wilder-Smith, A., \& Freedman, D. O. (2020). Isolation, quarantine, social distancing and community containment: pivotal role for old-style public health measures in the novel coronavirus (2019-nCoV) outbreak. Journal of travel medicine, 27(2).

\section{COMO CITAR ESTE ARTIGO:}

Cardoso, A. de O., Sousa, N. G., Cardoso, R. F., Utsumi, A. G. (2020). Análise de estratégias de isolamento social para o enfrentamento da pandemia da Covid-19 em Minas Gerais/Brasil. Holos. 36(5), 1-19.

\section{SOBRE OS AUTORES}

\section{A. DE O. CARDOSO}

Possui graduação em Engenharia Química pela Universidade Federal de Uberlândia (2009) e mestrado em Engenharia Química pela Universidade Federal de Uberlândia (2011) e doutorado pela Universidade de São Carlos (UFSCar). Atualmente é Professora Adjunta na Universidade Federal dos Vales do Jequitinhonha e Mucuri (UFVJM), atuando principalmente nos seguintes temas: Modelagem, Otimização e Controle de Processos Químicos com ênfase em sistemas não-lineares, controladores preditivos (MPC), otimização de sistemas em larga escala e plantwide control. E-mail: anamaria.cardoso@ict.ufvjm.edu.br ORCID ID: http://orcid.org/0000-0002-4518-075X

\section{N. G. SOUSA}

Possui graduação em Engenharia Química pela Universidade Federal de Uberlândia (2008). Mestrado em Engenharia Química pela Universidade Federal de Uberlândia (2010) com ênfase em propagação de falhas e Doutorado em Engenharia Química pela mesma instituição (2015) com ênfase em controle tolerante a falhas por alocação de controle. Tem experiência na área de Modelagem, Simulação, Otimização e Controle de Processos Químicos. Atualmente é docente da Universidade Federal do Triângulo Mineiro no curso de 
Engenharia Química. E-mail: nadia.sousa@uftm.edu.br

ORCID ID: http://orcid.org/0000-0002-3142-5875

\section{R. F. CARDOSO}

Possui graduação em Educação Física pela Universidade Federal de Uberlândia (2008). Pós graduado em acupuntura e mestrado em Reabilitação e Desempenho Funcional pela Universidade Federal dos Vales do Jequitinhonha e Mucuri. Atualmente é professor do Instituto de Pós-Graduação Unisaúde lecionando no Curso de Acupuntura. Diretor do Instituto Lucano, onde ministra cursos da área da Medicina Tradicional Chinesa e práticas integrativas. Tem experiência na área de Educação Física e em ACUPUNTURA, atuando principalmente nos seguintes temas: qualidade de vida relacionada a saúde, qualidade de vida, dor e acupuntura. E-mail: renatofleuryc@gmail.com

ORCID ID: http://orcid.org/0000-0002-7724-1553

\section{A. G. UTSUMI}

Possui graduação em Engenharia Ambiental e mestrado em Ciências Cartográficas pela Universidade Estadual Paulista Júlio de Mesquita Filho, câmpus de Presidente Prudente. Doutor pelo programa de PósGraduação em Agronomia, área de concentração Ciência do Solo da Faculdade de Ciências Agrárias e Veterinárias -UNESP, Jaboticabal. Atualmente é Professor Adjunto da Universidade Federal do Triângulo Mineiro- UFTM, vinculado ao departamento de Engenharia Ambiental. Tem experiência nas áreas de Sistemas de Informações Geográficas, Cartografia e Sensoriamento Remoto, atuando principalmente nos seguintes temas: Análise de Imagens Baseada em Objetos Geográficos, análise temporal dos recursos naturais e classificação de imagens multiespectrais. E-mail: alex.utsumi@ uftm.edu.br

ORCID ID: http://orcid.org/0000-0002-8955-6050

Editor(a) Responsável: Leandro Costa

Pareceristas Ad Hoc: DÊNIS EMANUEL DA COSTA VARGAS E SILDENIR RIBEIRO

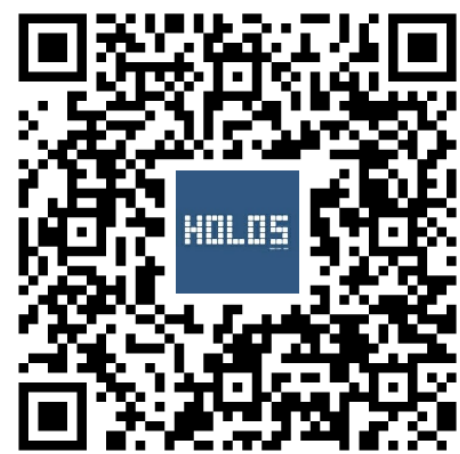

